\title{
Granular flows down inclined and vibrated planes: influence of basal friction
}

\author{
Naïma Gaudel ${ }^{\star}$, Sébastien Kiesgen de Richter ${ }^{\star}$, Nicolas Louvet, Mathieu Jenny, and Salaheddine Skali-Lami \\ ${ }^{1}$ Laboratoire d'Energétique et de Mécanique Théorique et Appliquée (LEMTA), Université de Lorraine-CNRS, UMR 7563, \\ Vandœuvre-lès-Nancy, 54504, France
}

\begin{abstract}
We present an experimental study about granular avalanches when external mechanical vibrations are applied. The results of the flow properties highlight the existence of two distinct regimes: (i) a gravity-driven regime at large angles where scaling laws are in agreement with those reported in the literature for non-vibrating granular flows and (ii) a vibration-driven regime at small angles where no flow occurs without applied vibrations. The flow in this regime is well described by a vibrationinduced activated process. We also propose an empirical law to capture the evolution of the thickness of the deposits as a function of the vibration intensity and the inclination angle.
\end{abstract}

\section{Introduction}

Free surface flows of granular matters down inclined planes are often encountered in geophysical situations, engineering applications or pharmaceutical manufactures. Vibratory solutions are often used in these industries to control the transport, the storage, or the mixing properties of the granular materials. Recent works have focused on the influence of vibrations on the rheology of granular flows in confined configurations [1-4]. The present work focuses on free surface flows properties of a beads assembly when both vibrations and gravity act, and on the influence of the basal friction.

\section{Experimental methods}

Our experimental setup is sketched in Fig. 1. Grains are released from a tank of volume $900 \mathrm{~cm}^{3}$ and from an adjustable height $\delta$. Experiments are performed for four different beads systems (Table 1). Samples are forced by transverse sinusoidal vibrations that we quantify through the relative acceleration $\Gamma=A(2 \pi f)^{2} / g$, where $g$ is gravity, $f$ is the frequency and $A$ the amplitude of vibrations. A CCD camera recording the flow is placed above the plane, at $25 \mathrm{~cm}$ from the aperture. The particle image velocimetry (PIV) provides us surface velocity $u_{s}$ measurements. The beads fall inside a reservoir resting on a scale: this provides us mean flow velocities $\bar{u}$ measurements. The local flow height $h$ is measured using the deviation of a laser sheet inclined at a small angle of incidence $\left(\approx 3^{\circ}\right)$ over the layer. The influence of $\theta, \Gamma$, and $d / \lambda$ on the flows are studied.

^e-mail: naima.gaudel@univ-lorraine.fr

${ }^{\star}$ e-mail: sebastien.kiesgen@univ-lorraine.fr

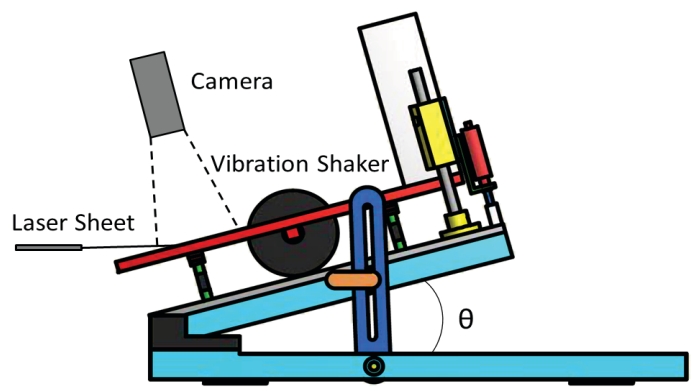

Figure 1. Experimental setup of glass beads (diameter $d$, $\rho \approx 2500 \mathrm{~kg} / \mathrm{m}^{3}$ ) flowing down a $40-\mathrm{cm}$-long and $10-\mathrm{cm}$ wide plane, covered with glass beads of average roughness $\lambda$, that can be inclined from horizontal up to $\theta=35^{\circ}$.

Table 1. The four systems of bulk and glued beads studied.

\begin{tabular}{ccc}
\hline System & Bulk $(d)$ & Rough surface $(\lambda)$ \\
\hline \hline 1 & $120 \mu m \pm 30$ & $300 \mu m \pm 100$ \\
2 & $120 \mu m \pm 30$ & $500 \mu m \pm 100$ \\
3 & $300 \mu m \pm 100$ & $500 \mu m \pm 100$ \\
4 & $500 \mu m \pm 100$ & $300 \mu m \pm 100$ \\
\hline
\end{tabular}

\section{Experimental results and discussion}

\subsection{Existence of two regimes}

Regarding the stationarity, the quantities $h$ and $u_{s}$ remain constant after a short transient regime of $20 \mathrm{~s}$ duration. After that, the flow is considered steady, and $h$ and $u_{s}$ relax to $h_{\text {stop }}$ and zero value, respectively. The mean values $\bar{h}$ and $\bar{u}_{s}$ are calculated by averaging 


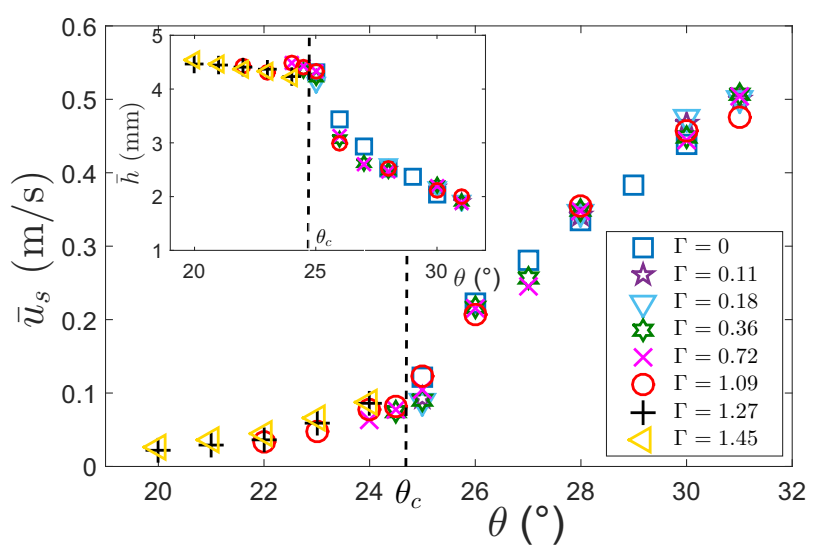

Figure 2. Evolution of $\bar{u}_{s}$ as a function of $\theta$ for the system $1(\delta=5 \mathrm{~mm})$ and different values of the $\Gamma$ number. In insert, the variation of $\bar{h}$ with $\theta$ for the same parameters. Two regimes appear.

these quantities over the steady-state regime. We define a critical angle $\theta_{c}$, for which there is no flow in the absence of vibrations.

Evolution of $\bar{u}_{s}$ and $\bar{h}$ as functions of $\theta$ have been plotted in Fig. 2 for the system 1 . The angle $\theta_{c}$ seems to be a transition between two distinct regimes. The fit of the variation of $\bar{u}_{s}$ with $\bar{h}$ leads to a power law behavior with an exponent $n=3 / 2 \pm 0.08$ for $\theta>\theta_{c}$. This result is in agreement with previous experimental and numerical studies [5, 6]. For $\theta<\theta_{c}$, the exponent takes a smaller value of $n=1 / 2 \pm 0.05$. It suggests that the flow dynamics flows down at small angles and confirms the existence of two regimes separated by a critical angle $\theta_{c}$ below which no flow occurs without vibrations:

- for $\theta>\theta_{c}, \bar{u}_{s}$ and $\bar{h}$ significantly vary when $\theta$ changes (high-angle regime),

- for $\theta<\theta_{c}, \bar{u}_{s}$ and $\bar{h}$ slightly vary when $\theta$ changes (low-angle regime).

We also study the flow structure along the plane ( $x$-direction) in this steady-state regime. We observed that $\bar{h}$ gradually decreases along the plane and finally reaches a constant value for $\theta>\theta_{c}$. This behavior has already been observed in granular flows without vibrations $[7,8]$. However, this phenomenon is nonexistent below the critical angle. A possible explanation is that flows are slow and homogenized by the vibrations for $\theta<\theta_{c}$, whereas they are heterogeneous due to gravity for $\theta>\theta_{c}$. To go through this limitation, a special care was taken to analyze the flow properties at a constant distance of $25 \mathrm{~cm}$ from the gate.

\subsection{Study of arrested flow}

The study of the variation of the deposits with the vibration intensity showed that $h_{\text {stop }}(\theta)$ is well controlled by $\Gamma$ for all $\theta<\theta_{c}$ and for a given angle when $\theta>\theta_{c}$ (Fig. 3). The value of $h_{\text {stop }}$ linearly decreases

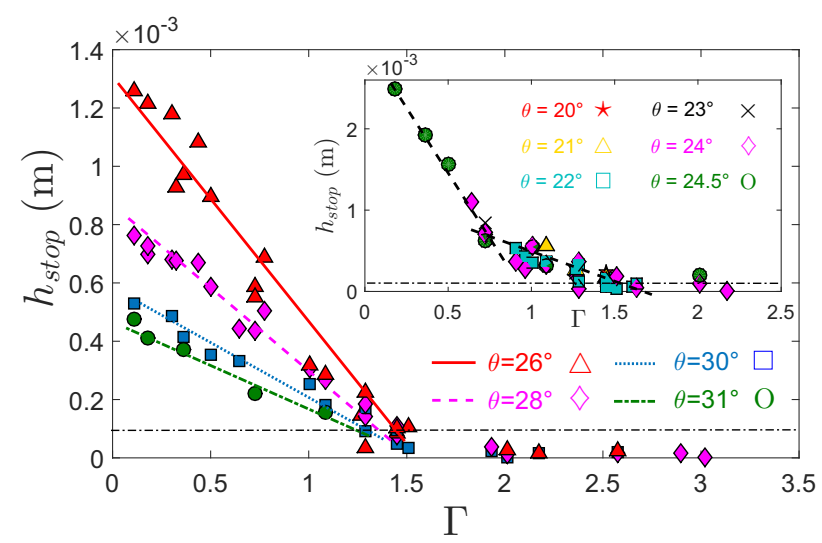

Figure 3. Evolution of $h_{\text {stop }}$ as a function of the $\Gamma$ number, for the system 1 , for $\theta>\theta_{c}$. In insert, $h_{\text {stop }}$ vs. $\Gamma$ for $\theta<\theta_{c}$. The black dash-dot lines corresponds to the limit $h_{\text {stop }}=d$ : below this, data values are similar to error measurements and are not taken into account.

with $\Gamma$ and vanishes at a critical value $\Gamma_{c}$ which appears to be quasi-constant for all $\theta$, in the range of values studied. This behavior has already been observed for the flow of a single bead down a transverse vibrated plane by Benedetti et al. [1]. We define $\Gamma_{c}$ as the critical relative acceleration for which $h_{\text {stop }}$ vanishes. To describe this dependency, we consider the flow as a three-layer flow: $h_{s t o p_{N V}}$ is the thickness of the deposits with no applied vibration, $h_{\text {stop }}$ the thickness of the deposits and $h$ the thickness of the flow. We define as $\left(h_{\text {stop }}-h_{\text {stop }}\right)$ the fluid thickness that flows due to vibrations. By analogy with the boundary layer for a Newtonian fluid [9] and by dimensional analysis, we consider $h_{s t o p_{N V}}$ as the penetration depth affected by vibrations and hypothesize that:

$$
h_{\text {stop }}=\kappa \times \sqrt{\frac{\mu_{a p p}}{\rho \omega}}
$$

here $v_{a p p}=\mu_{a p p} / \rho$ is the apparent viscosity (containing the dependence on $d / \lambda), \kappa$ is a coefficient with a value of 4.64 in the Newtonian case without vibrations, and $\omega=2 \pi f$. In that case, the stress can be written as $\tau_{s}=\rho g h_{\text {stop }_{N V}}$. We suppose that $\tau_{s}$ scales, at the first order, as:

$$
\tau_{s}=\mu_{a p p} \frac{A \omega}{h_{s t o p_{N V}}-h_{s t o p}}
$$

Combining these hypothesis, we can predict how vibrations modify the values of the deposits height:

$$
h_{\text {stop }}(\theta, \Gamma)=h_{s t o p_{N V}}(\theta)\left(1-\frac{\Gamma}{\kappa^{2}}\right)
$$

with $\kappa^{2}=\Gamma_{c}$ the critical value of the relative acceleration. The value of $\kappa$ is fixed at $1.20 \pm 0.2<4.64$ in experiments for the system 1 . This suggests that the value of $\Gamma_{c}$ is mainly controlled by rolling effects of the particles on the rough plane. 


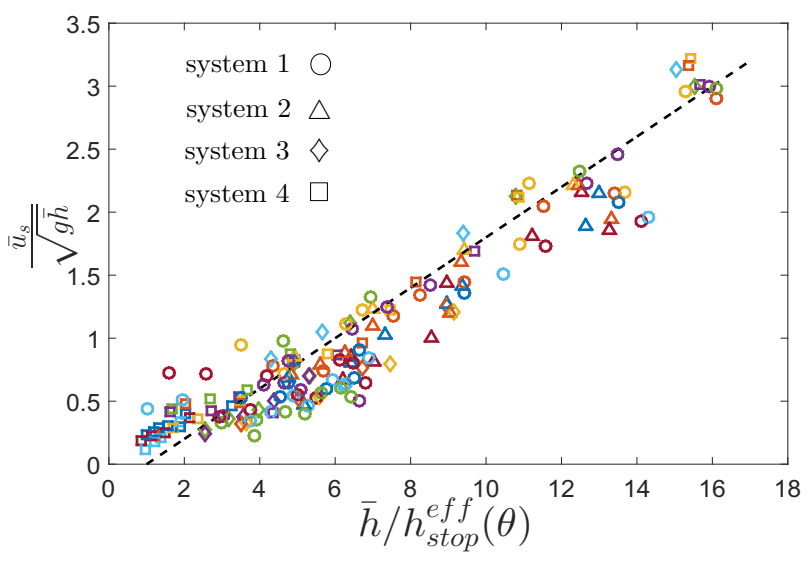

Figure 4. Evolution of the dimensionless velocity $\bar{u}_{s} / \sqrt{g \bar{h}}$ as a function of the rescaled thickness $\bar{h} / h_{\text {stop }}^{\text {eff }}(\theta)$ for the four systems studied (Tab. 1). Data are well fitted by this equation dotted line $\bar{u}_{s} / \sqrt{g \bar{h}}=0.2\left(\bar{h} / h_{\text {stop }}^{\text {eff }}(\theta)-1\right)$.

\subsection{Study of high-angle regime}

Previous studies have shown that the depth averaged velocity $\bar{u}$ is related to the thickness of the flow $\bar{h}$ in the steady-state regime by the flow rule [10-14]:

$$
\frac{\bar{u}}{\sqrt{g \bar{h}}}=\alpha+\beta \frac{\bar{h}}{h_{\text {stop }}(\theta)}
$$

where $g$ is gravity. This scaling law is valid when there is no vibrations and $h_{\text {stop }}(\theta)$ contains all the dependence in $\theta$ and $d / \lambda$. In the high-angle regime, measurements showed a proportionality of $\bar{u}$ with $\bar{u}_{s}$ such as $\bar{u} / \bar{u}_{s}=1 / 2 \pm 0.08$. This is not a Bagnold profile, suggesting that nonlocal effects are at play in our system $[11,12]$. The quantities $\bar{u}_{s}$ and $\bar{h}$ are not affected by vibrations for a fixed angle (Fig. 2). Thus, we replace $h_{\text {stop }}(\theta)$ with $h_{s t o p_{N V}}(\theta)$ in Eq. 4. The data then collapse into four different straight lines corresponding to the four systems: each of them is a particular relative roughness. In this study, the function $h_{s t o p_{N V}}(\theta)$ does not take into account the influence of $d / \lambda$. Therefore we propose to define a new stopping height:

$$
h_{\text {stop }}^{\text {eff }}(\theta)=h_{\text {stop }}(\theta) \frac{d}{\lambda}
$$

The data collapse into the same straight line (Fig. 4), the following scaling law seems to be suitable:

$$
F r=\frac{\bar{u}}{\sqrt{g \bar{h}}}=\tilde{\alpha}\left(\frac{\bar{h}}{h_{\text {stop }}^{e f f}}(\theta)-1\right)
$$

where the influence of the bead size $d$, the roughness condition $\lambda$ and the inclination angle $\theta$ is included in the single function $h_{\text {stop }}^{\text {eff }}(\theta)=h_{\text {stop }}(\theta) \frac{d}{\lambda}$, and $\tilde{\alpha}=0.1$ assuming $\bar{u} / \bar{u}_{s}=1 / 2$.

\subsection{Study of low-angle regime}

In the low-angle regime, flows do not occur without vibrations and $h_{\text {stop }}$ then diverges: the term $\bar{h} / h_{\text {stop }}(\theta)$
Table 2. The four systems studied and the corresponding values of the activation $\Gamma_{a}$ number.

\begin{tabular}{ccc}
\hline System & Relative roughness & $\Gamma_{a}$ \\
\hline \hline 1 & $1 / 3$ & $0.25 \pm 0.02$ \\
2 & $1 / 5$ & $0.35 \pm 0.03$ \\
3 & $3 / 5$ & $0.12 \pm 0.03$ \\
4 & $5 / 3$ & $0.08 \pm 0.03$ \\
\hline
\end{tabular}

in Eq. 4 tends to 0 . The only remaining contribution is the one: $\bar{u}=\tilde{\gamma} \sqrt{\bar{h}}$, with $\tilde{\gamma}=\alpha \sqrt{g}$. This is consistent with our experimental results that lead to a powerlaw evolution of $\bar{u}_{s}(\bar{h})$ with an exponent $n=1 / 2$ (see Sec. 3.1) and a ratio $\bar{u} / \bar{u}_{s}=2 / 3 \pm 0.1$. A minimum $\Gamma$ number, named $\Gamma_{a}$, is necessary to trigger the flow: for a given system, this value increases when $\theta$ decreases. The system needs more energy to break links between beads when the inclination decreases because the influence of gravity becomes lower. In the law-angle regime, vibrations impose the values of $\bar{u}_{s}$ and $h_{\text {stop }}(\theta)$. As expected, the scaling law (Eq. 4) does not describe the flow in our experiments. However, further investigations concerning the dependence of $\bar{u}_{s}$ on $h_{\text {stop }}$ reveal that $\bar{u}_{s} \propto \sqrt{\bar{h}} / h_{\text {stop }}^{2}(\theta)$ better rationalizes our experimental data. For a given $\Gamma$, the surface velocity linearly varies with $\sqrt{\bar{h}} / h_{\text {stop }}^{2}(\theta)$ (Fig. 5 , in insert). We expect a dependence of $\bar{u}_{s}$ with $\exp \left(-\frac{\Gamma}{\Gamma_{a}}\right)$, with $\Gamma_{a}$ the minimum relative acceleration necessary to trigger the flow. We reasonably assume that the flow can be described by a vibration induced activated process for which the dimensionless equation can be written as:

$$
\frac{\bar{u}_{s}}{\sqrt{g \bar{h}}}\left(\frac{h_{s t o p}}{d}\right)^{2} \propto \exp \left(-\frac{\Gamma}{\Gamma_{a}}\right)
$$

The semilog plot of $\bar{u}_{s} / \sqrt{g \bar{h}}$ as a function of $\Gamma$ provides us the value of $\Gamma_{a}$ for each system, using the slope value of $\Gamma_{a}$ (Tab. 2). The value of $\Gamma_{a}$ increases when $d / \lambda$ decreases because of the friction between beads [13]. Considering these values, Fig. 5 suggests that the scaling law (Eq. 7) correctly describes the flow in the low-angle regime. This exponential dependency shows that applying strong enough mechanical vibrations destabilizes the bulk structure. The probability for a grain to escape from a local trap raises with $\Gamma$ and $\theta$.

\section{Conclusions}

We studied granular avalanches down inclined and vibrated planes. Three results are highlighted:

(a) The existence of two flow regimes: a gravitydriven regime for $\theta>\theta_{c}$ and a vibration-driven regime for $\theta<\theta_{c}$.

(b) In the gravity-driven regime, vibrations only affect $h_{\text {stop }}$ and so the apparent flow rule $\mathrm{Fr}=$ 


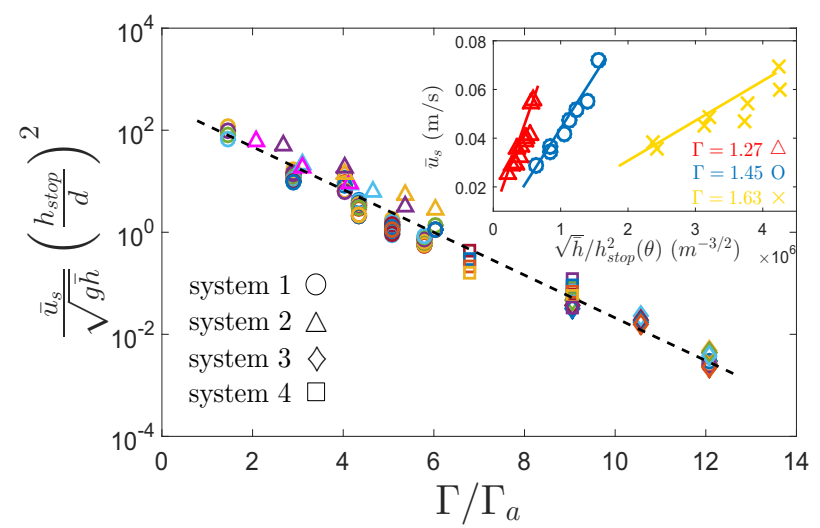

Figure 5. Semilog plot of $\bar{u}_{s} / \sqrt{g \bar{h}}$ as a function of $\Gamma / \Gamma_{a}$ for the four systems studied (Tab. 1). In insert, $\bar{u}_{s}$ vs. $\sqrt{\bar{h}} / h_{\text {stop }}^{2}(\theta)$ for the system 2 and different values of $\Gamma$.

$\tilde{\alpha}\left(\bar{h} / h_{\text {stop }}^{\text {eff }}(\theta)-1\right)$. We propose a linear dependency of $h_{\text {stop }}$ with $\Gamma$ such as $h_{\text {stop }}(\theta, \Gamma)=h_{\text {stop }}(\theta)\left(1-\Gamma / \Gamma_{c}\right)$.

(c) In the vibration-driven regime, flows occur due to vibrations. Increasing the relative roughness allow the minimum relative acceleration $\Gamma_{a}$ to decrease. Flows can be finely tuned by applying external vibrations. The scaling law in this regime is given by $\frac{\bar{u}_{s}}{\sqrt{g \bar{h}}}\left(\frac{h_{\text {stop }}}{d}\right)^{2} \propto \exp \left(-\frac{\Gamma}{\Gamma_{a}}\right)$.

\section{Acknowledgements}

The authors thank S. Becker, J. Bianchin, F. Demeurie, J.-Y. Morel for technical assistance and the students A. Pascot, M. Sandoungout, M. Stiti for their work.
This work is funded by European Founds Interreg VA (Project PowderReg).

\section{References}

[1] A. Benedetti, P. Sornay, B. Dalloz, M. Nicolas, Phys. Rev. E 85, 011307 (2012)

[2] C. Hanotin, S. Kiesgen de Richter, P. Marchal, L.J. Michot, C. Baravian, Phys. Rev. Lett. 108, 198301 (2012)

[3] C. Hanotin, S. Kiesgen de Richter, L.J. Michot, P. Marchal, Journal of Rheology 59, 253 (2015)

[4] C. Hanotin, P. Marchal, L.J. Michot, C. Baravian, S. Kiesgen de Richter, Soft Matter 9, 9352 (2013)

[5] J. Vallance, Ph.D. thesis, Michigan Technological University (1994)

[6] E. Azanza, Ph.D. thesis, Ecole Nationale des Ponts et Chaussees (1998)

[7] Y. Forterre, Ph.D. thesis, Universite de Provence - Aix-Marseille I (2002)

[8] C. Ancey, Physical Review E 65 (2001)

[9] H. Schlichting, K. Gersten, Boundary-layer theory (Springer Science \& Business Media, 2003)

[10] O. Pouliquen, Physics of Fluids 11, 542 (1999)

[11] S. Deboeuf, E. Lajeunesse, O. Dauchot, B. Andreotti, Physical review letters 97, 158303 (2006)

[12] F. Malloggi, B. Andreotti, E. Clement, Physical Review E 91 (2015)

[13] C. Goujon, Ph.D. thesis, Universite de Provence (2004)

[14] L.E. Silbert, J.W. Landry, G.S. Grest, Physics of Fluids 15, 1 (2003) 